\title{
On singular nonlinear distributional and impulsive initial and boundary value problems
}

\author{
Seppo Heikkilä
}

Correspondence: sheikki@cc.oulu.fi Department of Mathematical Sciences, University of Oulu, BOX 3000, FIN-90014, Oulu, Finland

\begin{abstract}
Purpose: To derive existence and comparison results for extremal solutions of nonlinear singular distributional initial value problems and boundary value problems.

Main methods: Fixed point results in ordered function spaces and recently introduced concepts of regulated and continuous primitive integrals of distributions. Maple programming is used to determine solutions of examples.

Results: New existence results are derived for the smallest and greatest solutions of considered problems. Novel results are derived for the dependence of solutions on the data. The obtained results are applied to impulsive differential equations. Concrete examples are presented and solved to illustrate the obtained results. MSC: 26A24, 26A39, 26A48, 34A12, 34A36, 37A37, 39B12, 39B22, 47B38, 47J25, 47H07, 47H10, 58D25
\end{abstract}

Keywords: distribution; primitive, integral; regulated, continuous; initial value problem, boundary value problem, singular, distributional

\section{Introduction}

In this paper, existence and comparison results are derived for the smallest and greatest solutions of first and second order singular nonlinear initial value problems as well as second order boundary value problems.

Recently, similar problems are studied in ordered Banach spaces, e.g., in [1-4], by converting problems into systems of integral equations, integrals in these systems being Bochner-Lebesgue or Henstock-Kurzweil integrals. A novel feature in the present study is that the right-hand sides of the considered differential equations comprise distributions on a compact real interval $[a, b]$. Every distribution is assumed to have a primitive in the space $\mathcal{R}[a, b]$ of those functions from $[a, b]$ to $\mathbb{R}$ which are left-continuous on $(a, b]$, right-continuous at $a$, and which have right limits at every point of $(a, b)$. With this presupposition, the considered problems can be transformed into integral equations which include the regulated primitive integral of distributions introduced recently in [5].

The paper is organized as follows. Distributions on $[a, b]$, their primitives, regulated primitive integrals and some of their properties, as well as a fixed point lemma are presented in Section 2. In Section 3, existence and comparison results are derived for the smallest and greatest solutions of first order initial value problems.

A fact that makes the solution space $\mathcal{R}[a, b]$ important in applications is that it contains primitives of Dirac delta distributions $\delta_{\lambda}, \lambda \in(a, b)$. This fact is exploited in

(c) 2011 Heikkilä; licensee Springer. This is an Open Access article distributed under the terms of the Creative Commons Attribution License (http://creativecommons.org/licenses/by/2.0), which permits unrestricted use, distribution, and reproduction in any medium, provided the original work is properly cited. 
Section 4, where results of Section 3 are applied to impulsive differential equations. The continuous primitive integral of distributions introduced in [6] is also used in these applications.

Existence of the smallest and greatest solutions of the second order initial and boundary value problems, and dependence of these solutions on the data are studied in Sections 5 and 6. Applications to impulsive problems are also presented.

Considered differential equations may be singular, distributional and impulsive. Differential equations, initial and boundary conditions and impulses may depend functionally on the unknown function and/or on its derivatives, and may contain discontinuous nonlinearities. Main tools are fixed point theorems in ordered spaces proved in [7] by generalized monotone iteration methods. Concrete problems are solved to illustrate obtained results. Iteration methods and Maple programming are used to determine solutions.

\section{Preliminaries}

Distributions on a compact real interval $[a, b]$ are (cf. [8]) continuous linear functionals on the topological vector space $\mathcal{D}$ of functions $\phi: \mathbb{R} \rightarrow \mathbb{R}$ possessing for every $j \in \mathbb{N}_{0}$ a continuous derivative $\phi^{(j)}$ of order $j$ that vanishes on $\mathbb{R} \backslash(a, b)$. The space $\mathcal{D}$ is endowed with the topology in which the sequence $\left(\phi_{k}\right)$ of $\mathcal{D}$ converges to $\varphi \in \mathcal{D}$ if and only if $\varphi_{k}^{(j)} \rightarrow \varphi^{(j)}$ uniformly on $(a, b)$ as $k \rightarrow \infty$ and $j \in \mathbb{N}_{0}$. As for the theory of distributions, see, e.g., $[9,10]$.

In this paper, every distribution $g$ on $[a, b]$ is assumed to have a primitive, i.e., a function $G \in \mathcal{R}[a, b]$ whose distributional derivative $G$ ' equals to $g$, in the function space

$$
\mathcal{R}[a, b]=\left\{G: \lim _{t \rightarrow s+} G(s) \text { exists, } \lim _{s \rightarrow t-} G(s)=G(t) \text { if } a \leq s<t \leq b, \text { and } G(a):=\lim _{s \rightarrow a+} G(s)\right\} .
$$

The value $\langle g, \phi\rangle$ of $g$ at $\varphi \in \mathcal{D}$ is thus given by

$$
\langle g, \varphi\rangle=\left\langle G^{\prime}, \varphi\right\rangle=-\left\langle G, \varphi^{\prime}\right\rangle=-\int_{a}^{b} G(t) \varphi^{\prime}(t) d t .
$$

Such a distribution $g$ is called $R P$ integrable. Its regulated primitive integral is defined by

$$
r \int_{s}^{t} g:=G(t)-G(s), \quad a \leq s \leq t \leq b, \quad \text { where } G \text { is a primitive of } g \text { in } \mathcal{R}[a, b] .
$$

As noticed in [5], the regulated primitive integral generalizes the wide Denjoy integral, and hence also Riemann, Lebesgue, Denjoy and Henstock-Kurzweil integrals.

Denote by $\mathcal{A}_{R}[a, b]$ the set of those distributions on $[a, b]$ that are $R P$ integrable on $[a, b]$. If $g \in \mathcal{A}_{R}[a, b]$, then the function $t \mapsto{ }^{r} \int_{a}^{t} g$ is that primitive of $g$ which belongs to the set

$$
\mathcal{P}_{R}[a, b]=\{G \in \mathcal{R}[a, b]: G(a)=0\} .
$$

It can be shown (cf. [5]) that a relation $\preccurlyeq$, defined by

$$
f \preccurlyeq g \text { in } \mathcal{A}_{R}[a, b] \text { if and only if } r \int_{a}^{t} f \leq^{r} \int_{a}^{t} g \text { for all } t \in[a, b],
$$


is a partial ordering on $\mathcal{A}_{R}[a, b]$. In particular,

$$
f=g \text { in } \mathcal{A}_{R}[a, b] \text { if and only if }{ }^{r} \int_{a}^{t} f=r \int_{a}^{t} g \text { for all } t \in[a, b] .
$$

Given partially ordered sets $X=(X, \leq)$ and $Y=(Y, \preccurlyeq)$, we say that a mapping $f: X \rightarrow$ $Y$ is increasing if $f(x) \leqslant f(y)$ whenever $x \leq y$ in $X$, and order-bounded if there exist $f_{ \pm} \in$ $Y$ such that $f_{-} \leqslant f(x) \preccurlyeq f_{+}$for all $x \in X$.

The following fixed point result is a consequence of [11], Theorem A.2.1, or [7], Theorem 1.2.1 and Proposition 1.2.1.

Lemma 2.1. Given a partially ordered set $P=(P, \leq)$, and its order interval $\left[x_{-}, x_{+}\right]=$ $\left\{x \in P: x_{-} \leq x \leq x_{+}\right\}$, assume that a mapping $G:\left[x_{-}, x_{+}\right] \rightarrow\left[x_{-}, x_{+}\right]$is increasing, and that each well-ordered chain of the range $G\left[x_{-}, x_{+}\right]$of $G$ has a supremum in $P$ and each inversely well-ordered chain of $G\left[x_{-}, x_{+}\right]$has an infimum in $P$. Then $G$ has the smallest and greatest fixed points, and they are increasing with respect to $G$.

Remarks 2.1. Under the hypotheses of Lemma 2.1, the smallest fixed point $x^{*}$ of $G$ is by [[7], Theorem 1.2.1] the maximum of the chain $C$ of $\left[x_{-}, x_{+}\right]$that is well ordered, i.e., every nonempty subset of $C$ has the smallest element, and that satisfies

(I) $x_{-}=\min C$, and if $x_{-}<x$, then $x \in C$ if and only if $x=\sup G[\{y \in C: y<x\}]$.

The smallest elements of $C$ are $G^{n}\left(x_{-}\right), n \in \mathbb{N}_{0}$, as long as $G^{n}\left(x_{-}\right)=G\left(G^{n-1}\left(x_{-}\right)\right)$is defined and $G^{n-1}\left(x_{-}\right)<G^{n}\left(x_{-}\right), n \in \mathbb{N}$. If $G^{n-1}\left(x_{-}\right)=G^{n}\left(x_{-}\right)$for some $n \in \mathbb{N}$, there is the smallest such an $n$, and $x_{*}=G^{n-1}\left(x_{-}\right)$is the smallest fixed point of $G$ in $\left[x_{-}, x_{+}\right]$. If $x_{\omega}=\sup _{n \in \mathbb{N}} G^{n}\left(x_{-}\right)$is defined in $P$ and is a strict upper bound of $\left\{G^{n}\left(x_{-}\right)\right\}_{n \in \mathbb{N}}$, then $x_{\omega}$ is the next element of $C$. If $x_{\omega}=G\left(x_{\omega}\right)$, then $x_{*}=x_{\omega}$, otherwise the next elements of $C$ are of the form $G^{n}\left(x_{\omega}\right), n \in \mathbb{N}$, and so on.

The greatest fixed point $x^{*}$ of $G$ is the minimum of the chain $D$ of $\left[x_{-}, x_{+}\right]$that is inversely well ordered, i.e., every nonempty subset of $D$ has the greatest element, and that has the following property:

(II) $x_{+}=\max D$, and if $x<x_{+}$, then $x \in D$ if and only if $x=\operatorname{infG}[\{y \in D: x<y\}]$.

The greatest elements of $D$ are $n$-fold iterates $G^{n}\left(x_{+}\right)$, as long as they are defined and $G^{n}\left(x_{+}\right)<G^{n-1}\left(x_{+}\right)$. If equality holds for some $n \in \mathbb{N}$, then $x^{*}=G^{n-1}\left(x_{+}\right)$is the greatest fixed point of $G$ in $\left[x_{-}, x_{+}\right]$.

\section{First order initial value problems}

In this section, existence and comparison results are derived for the smallest and greatest solutions of first order initial value problems. Denote by $L_{l o c}^{1}(a, b],-\infty<a<b<\infty$, the space of locally Lebesgue integrable functions from the half-open interval $(a, b]$ to R. $L_{l o c}^{1}(a, b]$ is ordered a.e. pointwise, and its a.e. equal functions are identified.

Given $p:[a, b] \rightarrow \mathbb{R}_{+}$, consider the initial value problem (IVP)

$$
(p \cdot u)^{\prime}=g(u), \quad \lim _{t \rightarrow a+}(p \cdot u)(t)=c(u),
$$

where $c(u) \in \mathbb{R}$, and $g(u) \in \mathcal{A}_{R}[a, b]$. We are looking for solutions of (3.1) from the set

$$
S=\left\{u \in L_{l o c}^{1}(a, b]: \lim _{t \rightarrow a+}(p \cdot u)(t) \text { exists, and } p \cdot u \in \mathcal{R}[a, b]\right\}
$$


Definition 3.1. We say that a function $u \in S$ is a subsolution of the IVP (3.1) if

$$
(p \cdot u)^{\prime} \preccurlyeq g(u) \text {, and } \lim _{t \rightarrow a+}(p \cdot u)(t) \leq c(u) .
$$

If reversed inequalities hold in (3.3), we say that $u$ is a supersolution of (3.1). If equalities hold in (3.3), then $u$ is called a solution of (3.1).

We shall first transform the IVP (3.1) into an integral equation.

Lemma 3.1. Given $c(u) \in \mathbb{R}, u \in L_{l o c}^{1}(a, b]$ and $p:[a, b] \rightarrow \mathbb{R}_{+}$, assume that $\frac{1}{p} \in L_{l o c}^{1}(a, b]$, and that $g(u) \in \mathcal{A}_{R}[a, b]$. Then $u$ is a solution of the IVP (3.1) in $S$ if and only if $u$ is a solution of the following integral equation:

$$
u(t)=\frac{1}{p(t)}\left(c(u)+r \int_{a}^{t} g(u)\right), \quad t \in(a, b] .
$$

Proof: Assume that $u$ is a solution of (3.1) in $S$. The definition of $S$ and (3.1) ensure by (2.2) that

$$
r \int_{r}^{t} g(u)=r \int_{r}^{t}(p \cdot u)^{\prime}=(p \cdot u)(t)-(p \cdot u)(r), \quad a<r \leq t<b .
$$

Allowing $r$ tend to $a+$ and applying the initial condition of (3.1) we see that (3.4) is valid. Conversely, let $u$ be a solution of (3.4). According to (3.4) we have

$$
(p \cdot u)(t)=c(u)+r \int_{a}^{t} g(u), \quad t \in(a, b] .
$$

This equation implies that $u \in S$, that the initial condition of (3.1) is valid, and that

$$
(p \cdot u)^{\prime}=g(u) \text {. }
$$

Thus, $u$ is a solution of the IVP (3.1) in $S$.

Our first existence and comparison result for the IVP (3.1) reads as follows.

Theorem 3.1. Assume that $g: L_{l o c}^{1}(a, b] \rightarrow \mathcal{A}_{R}[a, b]$ is increasing, that $p:[a, b] \rightarrow \mathbb{R}_{+}$, that $\frac{1}{p} \in L_{l o c}^{1}(a, b]$, and that the IVP (3.1) has a subsolution $u_{-}$and a supersolution $u_{+}$in $S$ satisfying $u_{-} \leq u_{+}$. Then (3.1) has the smallest and greatest solutions within the order interval $\left[u_{-}, u_{+}\right]$of $S$. Moreover, these solutions are increasing with respect to $g$ and $c$.

Proof: Define a mapping $G: L_{l o c}^{1}(a, b] \rightarrow L_{l o c}^{1}(a, b]$ by

$$
G(u)(t):=\frac{1}{p(t)}\left(c(u)+r \int_{a}^{t} g(u)\right), \quad t \in(a, b] .
$$

Because $g$ is increasing, it follows from (2.3) and (3.6) that $G$ is increasing. Applying (2.3), [[5], Theorem 7] and Definition 3.1 we see that if $u \in L_{l o c}^{1}(a, b]$ and $u_{-} \leq u \leq u_{+}$, then

$$
\begin{aligned}
\left(p \cdot u_{-}\right)(t)-c(u) & \leq\left(p \cdot u_{-}\right)(t)-\lim _{r \rightarrow a_{+}}\left(p \cdot u_{-}\right)(r)=\lim _{r \rightarrow a_{+}} r \int_{r}^{t}\left(p \cdot u_{-}\right)^{\prime} \\
& =r \int_{a}^{t}\left(p \cdot u_{-}\right)^{\prime} \leq r \int_{a}^{t} g\left(u_{-}\right) \leq r \int_{a}^{t} g(u), \quad a<t \leq b .
\end{aligned}
$$


Thus

$$
u_{-}(t) \leq \frac{1}{p(t)}\left(c(u)+r \int_{a}^{t} g(u)\right)=G(u)(t), \quad t \in(a, b] .
$$

Similarly, it can be shown that $G(u)(t) \leq u_{+}(t)$ for each $t \in(a, b]$. Thus, $G$ maps the order interval $\left[u_{-}, u_{+}\right]$of $L_{l o c}^{1}(a, b]$ into $\left[u_{-}, u_{+}\right]$. Let $W$ be a well-ordered or an inversely well-ordered chain in $G\left[u_{-}, u_{+}\right]$. It follows from [[1], Proposition 9.36] and its dual that sup $W$ and inf $W$ exist in $L_{l o c}^{1}(a, b]$.

The above proof shows that the operator $G$ defined by (3.6) satisfies the hypotheses of Lemma 2.1 when $P=L_{l o c}^{1}(a, b]$. Thus $G$ has the smallest fixed point $u_{*}$ and the greatest fixed point $u^{*}$ in $\left[u_{-}, u_{+}\right]$. These fixed points are the smallest and greatest solutions of the integral equation (3.4) in $\left[u_{-}, u_{+}\right]$. This result and Lemma 3.1 imply that $u_{*}$ and $u^{*}$ belong to $S$, and they are the smallest and greatest solutions of the IVP (3.1) in $\left[u_{-}, u_{+}\right]$. Moreover, $u_{*}$ and $u^{*}$ are by Lemma 2.1 increasing with respect to $G$. This result implies by (2.3) and (3.6) the last conclusion of Theorem.

The following result is a consequence of Theorem 3.1.

Proposition 3.1. Assume that mappings $g: L_{l o c}^{1}(a, b] \rightarrow \mathcal{A}_{R}[a, b]$ and $c: L_{l o c}^{1}(a, b] \rightarrow \mathbb{R}$ are increasing and order-bounded, that $p:[a, b] \rightarrow \mathbb{R}_{+}$, and that $\frac{1}{p} \in L_{l o c}^{1}(a, b]$. Then, the IVP (3.1) has in $S$ the smallest and greatest solutions that are increasing with respect to $g$ and $c$.

Proof: Because $g$ and $c$ are order-bounded, there exist $g_{ \pm} \in \mathcal{A}_{R}[a, b]$ and $c_{ \pm} \in \mathbb{R}$ such that $g \leqslant g(x) \leqslant g_{+}$and $c_{-} \leq c(x) \leq c_{+}$for all $x \in L_{l o c}^{1}(a, b]$. Denote

$$
u_{ \pm}(t)=\frac{1}{p(t)}\left(c_{ \pm}{ }^{r} \int_{a}^{t} g_{ \pm}\right), \quad t \in(a, b]
$$

Then $u_{ \pm} \in S$, and

$$
\left(p \cdot u_{-}\right)^{\prime}=g_{-} \preccurlyeq g(x) \preccurlyeq g_{+}=\left(p \cdot u_{+}\right)^{\prime} \text { for all } x \in L_{l o c}^{1}(a, b],
$$

and

$$
\lim _{t \rightarrow a+}\left(p \cdot u_{-}\right)(t)=c_{-} \leq c(x) \leq c_{+}=\lim _{t \rightarrow a_{+}}\left(p \cdot u_{+}\right) \quad \text { for all } x \in L_{l o c}^{1}(a, b] .
$$

Thus $u_{-}$is a subsolution and $u_{+}$is a supersolution of (3.1), whence the IVP (3.1) has by Theorem 3.1 the smallest solution $u_{*}$ and the greatest solution $u^{*}$ in the order interval $\left[u_{-}, u_{+}\right]$of $S$.

If $u \in S$ is any solution of (3.1), then

$$
\frac{1}{p(t)}\left(c_{-}+r \int_{a}^{t} g_{-}\right) \leq \frac{1}{p(t)}\left(c(u)+r \int_{a}^{t} g(u)\right) \leq \frac{1}{p(t)}\left(c_{+}+{ }^{r} \int_{a}^{t} g_{+}\right), \quad t \in(a, b],
$$

or equivalently,

$$
u_{-}(t) \leq u(t) \leq u_{+}(t), \quad t \in(a, b] .
$$

Consequently, $u \in\left[u_{-}, u_{+}\right]$, whence $u_{*}$ and $u^{*}$ are the smallest and greatest of all the solutions of (3.1) in $S$.

In the next proposition, the Henstock-Kurzweil integral $K \int$ can be replaced by any of the integrals called Riemann, Lebesgue, Denjoy and wide Denjoy integrals. 
Proposition 3.2. Assume that $g(x)$ is RP integrable on $[a, b]$ for every $x \in L_{l o c}^{1}(a, b]$, and that

$$
r \int_{a}^{t} g(x)=\sum_{i=1}^{n} H_{i}(t)^{K} \int_{a}^{t} f_{i}(x)+H_{0}(t), \quad t \in[a, b]
$$

Where $H_{0} \in \mathcal{P}_{R}[a, b]$, and for each $i=1, \ldots, n, H_{i}:[a, b] \rightarrow[0, \infty)$ has right limits on $[a, b)$, is left-continuous on $(a, b]$, and $f_{i}:[a, b] \rightarrow \mathbb{R}$ satisfies the following hypotheses.

$\left(\mathbf{f}_{i 1}\right) f_{i}(x)$ is Henstock-Kurzweil integrable on $[a, b]$ for every $x \in L_{\text {loc }}^{1}(a, b]$.

$\left(\mathbf{f}_{i 2}\right)$ There exist Henstock-Kurzweil integrable functions $\underline{f}_{i}, \bar{f}_{i}:[a, b] \rightarrow \mathbb{R}$ such that ${ }^{K} \int_{a}^{t} f_{-i} \leq{ }^{K} \int_{a}^{t} f_{i}(x) \leq{ }^{K} \int_{a}^{t} f_{i}(y) \leq{ }^{K} \int_{a}^{t} \bar{f}_{i,} t \in[a, b]$ whenever $x \leq y$ in $L_{l o c}^{1}(a, b]$.

If $c: L_{l o c}^{1}(a, b] \rightarrow \mathbb{R}$ is increasing and order-bounded, then the IVP (3.1) has in $S$ the smallest and greatest solutions that are increasing with respect to $f_{i}$ and $c$.

Proof: The hypotheses imposed above ensure by (2.3) and (3.7) that $g$ is an increasing mapping from $L_{l o c}^{1}(a, b]$ to the order interval $\left[g_{-}, g_{+}\right]$of $\mathcal{A}_{R}[a, b]$, where

$$
r \int_{a}^{t} g_{-}=\sum_{i=1}^{n} H_{i}(t) K \int_{a}^{t} f_{-i}+H_{0}(t), \quad r \int_{a}^{t} g_{+}=\sum_{i=1}^{n} H_{i}(t){ }^{K} \int_{a}^{t} \bar{f}_{i}+H_{0}(t), \quad t \in[a, b] .
$$

Thus the conclusions follow from Proposition 3.1.

Example 3.1. Assume that

$$
r \int_{a}^{t} g(x)=H_{1}(t)^{K} \int_{a}^{t} f_{1}(x)+H_{0}(t), \quad t \in[0, b],
$$

where $b \geq 1, \quad H_{0} \in \mathcal{R}[0, b], \quad H_{1}$ is the Heaviside step function, i.e., $\left\{\begin{array}{l}f_{1}(x)(t)=\frac{1}{10^{5}}\left[10^{5} \arctan \left(\int_{1 / 2}^{1}\left(x(t)-\frac{H_{0}(t)}{p(t)}\right) d t\right)\right]\left(\left|\sin \left(\frac{1}{t}\right)\right|-\frac{1}{t} \operatorname{sgn}\left(\sin \left(\frac{1}{t}\right)\right) \cos \left(\frac{1}{t}\right)\right), \\ t \in(0, b], x \in L_{l o c}^{1}(0, b],[z]=\max \{n \in \mathbb{Z}: n \leq z\} \text { and } \operatorname{sgn}(z)= \begin{cases}z /|z|, & z \neq 0, \\ 0, & z=0 .\end{cases} \end{array}\right.$

$$
\left\{\begin{array}{l}
f_{1}(x)(t)=\frac{1}{10^{5}}\left[10^{5} \arctan \left(\int_{1 / 2}^{1}\left(x(t)-\frac{H_{0}(t)}{p(t)}\right) d t\right)\right]\left(\left|\sin \left(\frac{1}{t}\right)\right|-\frac{1}{t} \operatorname{sgn}\left(\sin \left(\frac{1}{t}\right)\right) \cos \left(\frac{1}{t}\right)\right), \\
t \in(0, b], x \in L_{l o c}^{1}(0, b],[z]=\max \{n \in \mathbb{Z}: n \leq z\} \text { and } \operatorname{sgn}(z)= \begin{cases}z /|z|, & z \neq 0, \\
0, & z=0 .\end{cases}
\end{array}\right.
$$

Note, that the greatest integer function [.] occurs in the function $f_{1}(x)$. Prove that the IVP

$$
(p \cdot u)^{\prime}=g(u), \quad \lim _{t \rightarrow 0+}(p \cdot u)(t)=0,
$$

where $p(t)=t, t \in[0, b]$, has the smallest and greatest solutions, and calculate them.

Solution: Problem (3.9) is of the form (3.1), where $c(u)=a=0$ and $p(t) \equiv t$. The hypotheses $\left(f_{11}\right)$ and $\left(f_{21}\right)$ are valid when

$$
\begin{aligned}
& K \int_{0}^{t} f_{-_{1}}=-2 t\left|\sin \left(\frac{1}{t}\right)\right|+H_{0}(t), \quad t \in(0, b], \\
& K \int_{0}^{t} \bar{f}_{1}=2 t\left|\sin \left(\frac{1}{t}\right)\right|+H_{0}(t), \quad t \in(0, b] .
\end{aligned}
$$


Thus the IVP (3.9) has by Proposition 3.2 the smallest and greatest solutions. They are the smallest and greatest fixed points of the mapping $G$ defined by

$$
G(x)(t):=\frac{1}{t} r \int_{0}^{t} g(x), \quad t \in(0, b], x \in L_{l o c}^{1}(0, b] .
$$

$G$ is an increasing mapping from $L_{l o c}^{1}(0, b]$, to its order interval $\left[u_{-}, u_{+}\right]$, where

$$
u_{ \pm}(t):= \pm 2\left|\sin \left(\frac{1}{t}\right)\right|+\frac{H_{0}(t)}{t}, \quad t \in(0, b] .
$$

Calculating the successive approximations $G^{n}\left(u_{ \pm}\right)$we see that $G^{7}\left(u_{ \pm}\right)=G^{8}\left(u_{ \pm}\right)$. This means by Remark 2.1 that $u_{*}=G^{7}\left(u_{-}\right)$and $u^{*}=G^{7}\left(u_{+}\right)$are the smallest and greatest fixed points of $G$ in $\left[u_{-}, u_{+}\right]$. According to the proof of Proposition 3.1, $u_{*}$ and $u^{*}$ are also the smallest and greatest solutions, of the initial value problem (3.9) in $S$. The exact expressions of $u_{*}$ and $u^{*}$ are:

$$
\left\{\begin{array}{l}
u_{*}(t)=-\arctan \left(\frac{67229}{10000}\right)\left|\sin \left(\frac{1}{t}\right)\right|+\frac{H_{0}(t)}{t}, \quad t \in(0, b], \\
u^{*}(t)=\arctan \left(\frac{16807}{2500}\right)\left|\sin \left(\frac{1}{t}\right)\right|+\frac{H_{0}(t)}{t}, \quad t \in(0, b] .
\end{array}\right.
$$

\section{Applications to impulsive problems}

In this section, we assume that $\Lambda$ is a well-ordered subset of $(a, b)$. Let $\delta_{\lambda}, \lambda \in \Lambda$, denote the translation of Dirac delta distribution for which ${ }^{r} \int_{a}^{t} \delta_{\lambda}=H(t-\lambda), t \geq a$, where $H$ is the Heaviside step function. Consider the singular distributional Cauchy problem

$$
(p \cdot u)^{\prime}=\sum_{\lambda \in \Lambda} I(\lambda, u) \delta_{\lambda}+f(u), \quad \lim _{t \rightarrow a_{+}}(p \cdot u)=c(u),
$$

where $p:[a, b] \rightarrow \mathbb{R}_{+}$and $\frac{1}{p} \in L_{l o c}^{1}(a, b]$. The values of $f$ are distributions on $[a, b]$, and the values of $I$ are real numbers.

Definition 4.1. By a solution of (4.1), we mean such a function $u \in S$ that satisfies (4.1), for which $p \cdot u$ is continuous on $[a, b] \backslash \Lambda$, and has impulses

$$
\Delta(p \cdot u)(\lambda):=(p \cdot u)(\lambda+)-(p \cdot u)(\lambda)=I(\lambda, u), \lambda \in \Lambda .
$$

In the study of (4.1), the regulated primitive integral is replaced by the continuous primitive integral presented in [6]. A distribution $g$ on $[a, b]$ is called distributionally Denjoy $(D D)$ integrable on $[a, b]$, denote $g \in \mathcal{A}_{C}[a, b]$, if $g$ has a continuous primitive, i.e., $g$ is a distributional derivative of a function $G \in C[a, b]$. The continuous primitive integral of $g$ is defined by

$$
c \int_{s}^{t} g=G(t)-G(s), \quad a \leq s \leq t \leq b .
$$

$\mathcal{A}_{C}[a, b]$ is a proper subset of $\mathcal{A}_{R}[a, b]$, and for every $g \in \mathcal{A}_{C}[a, b]$ its continuous and regulated primitive integrals are equal. As shown in [6], $\mathcal{A}_{C}[a, b]$ contains functions that are wide Denjoy integrable, and hence also Riemann, Lebesgue, Denjoy and HenstockKurzweil integrable on $[a, b]$. On the other hand, distributional derivatives of nowhere differentiable Weierstrass function and almost everywhere differentiable Cantor function are distributionally but not wide Denjoy integrable. 
It can be shown (cf. [6]) that relation $\preccurlyeq$, defined by

$$
f \preccurlyeq g \text { if and only if } \int_{a}^{t} f \leq^{c} \int_{a}^{t} g \text { for all } t \in[a, b],
$$

is a partial ordering on $\mathcal{A}_{C}[a, b]$.

Transformation of the Cauchy problem (4.1) into an integral equation is presented in the following lemma.

Lemma 4.1. Assume that $u \in S$, that $f(u) \in \mathcal{A}_{C}[a, b]$, and that $\sum_{\lambda \in \Lambda}|I(\lambda, u)|<\infty$.

Then $u$ is a solution of (4.1) if and only if

$$
u(t)=\frac{1}{p(t)}\left(c(u)+\sum_{\lambda \in \Lambda} I(\lambda, u) H(t-\lambda)+{ }^{c} \int_{a}^{t} f(u)\right), \quad t \in(a, b] .
$$

Proof: Assume first that $u \in S$ satisfies (4.3). Because $\Lambda$ is well-ordered, it follows that if $\lambda \in \Lambda$ and $\lambda<\sup \Lambda$, then $H(t-\lambda)=1$ on $(\lambda, S(\lambda)$ ], where $S(\lambda)=\min \{\mu \in \Lambda$ : $\lambda<\mu\}$. This property implies that if the function $v:(a, b] \rightarrow \mathbb{R}$ is defined by

$$
v(t)=\frac{1}{p(t)}\left(c(u)+\sum_{\lambda \in \Lambda} I(\lambda, u) H(t-\lambda)\right), \quad t \in(a, b],
$$

then the function $p \cdot v$ is constant on every interval $(\lambda, S(\lambda)], \Lambda \ni \Lambda<\sup \Lambda$, on [a, $\min \Lambda]$, and on $(\sup \Lambda, b]$ if sup $\Lambda<b$. In particular, $p \cdot v \in \mathcal{R}[a, b]$, and the distributional derivative of $p \cdot v$ is

$$
(p \cdot v)^{\prime}=\sum_{\lambda \in \Lambda} I(\lambda, u) \delta_{\lambda}
$$

Thus

$$
(p \cdot u)^{\prime}=(p \cdot v)^{\prime}+f(u)=\sum_{\lambda \in \Lambda} I(\lambda, u) \delta_{\lambda}+f(u) .
$$

Since $t \mapsto{ }^{c} \int_{a}^{t} f(u)$ is continuous on $[a, b]$, then $p \cdot u$ is continuous on $[a, b] \backslash \Lambda$. Because

$$
(p \cdot v)(t)-(p \cdot v)(\lambda)=I(\lambda, u) H(t-\lambda)=I(\lambda, u), \quad \lambda \in \Lambda, t \in(\lambda, S(\lambda)],
$$

then

$$
\Delta(p \cdot u)(\lambda)=(p \cdot u)(\lambda+)-(p \cdot u)(\lambda)=(p \cdot v)(\lambda+)-(p \cdot v)(\lambda)=I(\lambda, u), \quad \lambda \in \Lambda .
$$

Moreover $\lim _{t \rightarrow a_{+}}(p \cdot u)(t)=c(u)$, so that $u$ is a solution of the IVP (4.1).

Assume next that $u \in S$ is a solution of (4.1). Denoting

$$
z(t)=u(t)-v(t), \quad t \in[a, b],
$$

where $v$ is defined by (4.4), it follows from (4.1) and (4.5) that

$$
(p \cdot z)^{\prime}=f(u), \quad \lim _{t \rightarrow a+}(p \cdot z)=0 .
$$

Because $f(u)$ is $D D$ integrable on $[a, b]$, then

$$
(p \cdot z)(t)={ }^{c} \int_{a}^{t} f(u), \quad t \in[a, b]
$$


Thus

$$
(p \cdot u)(t)=(p \cdot z)(t)+(p \cdot v)(t)=c(u)+\sum_{\lambda \in \Lambda} I(\lambda, u) H(t-\lambda)+{ }^{c} \int_{a}^{t} f(u), \quad t \in[a, b]
$$

or equivalently, (4.3) holds. $\square$

Noticing that the IVP (4.1) is a special case of the Cauchy problem (3.1), where

$$
g(u)=\sum_{\lambda \in \Lambda} I(\lambda, u) \delta_{\lambda}+f(u)
$$

the results of Section 3 can be applied to study the IVP (4.1). The following result is a consequence of Proposition 3.1.

Proposition 4.1. The distributional IVP (4.1) has the smallest and greatest solutions that are increasing with respect to $f$ and $c$, if $f: L_{l o c}^{1}(a, b] \rightarrow \mathcal{A}_{C}[a, b]$ and $c: L_{l o c}^{1}(a, b] \rightarrow \mathbb{R}$ are increasing and order-bounded, if $p:[a, b] \rightarrow \mathbb{R}_{+}$, if $\frac{1}{p} \in L_{l o c}^{1}(a, b]$, and if $I: \Lambda \times L_{\text {loc }}^{1}(a, b] \rightarrow \mathbb{R}$ has the following properties.

(I) $\sum_{\lambda \in \Lambda}|I(\lambda, x)| \leq M<\infty$ for all $x \in L_{\text {loc }}^{1}(a, b]$, and $x \mapsto I(\lambda, x)$ is increasing when $\lambda \in$ $\Lambda$.

Proof: The given hypotheses imply that (4.6) defines a mapping $g: L_{\text {loc }}^{1}(a, b] \rightarrow \mathcal{A}_{R}[a, b]$ that is increasing and order-bounded. Thus, the IVP (3.1) has by Proposition 3.1 the smallest solution $u_{*}$ and the greatest solution $u^{*}$ in $S$, and they are increasing with respect to $g$ and $c$. By Lemma 4.1, $u_{*}$ and $u^{*}$ are the smallest and greatest solutions of the IVP (4.1), and they are increasing with respect to $f$, and $c$, since $g$ is increasing with respect to $f$. $\square$

The initial value problem

$$
\frac{d}{d t}(p(t) u(t))=q(t, u(t), u) \text { a.e. on }[a, b], \quad \lim _{t \rightarrow a+}(p(t) u(t))=c(u),
$$

combined with the impulsive property:

$$
\Delta(p \cdot u)(\lambda)=(p \cdot u)(\lambda+)-(p \cdot u)(\lambda)=I(\lambda, u), \quad \lambda \in \Lambda,
$$

form a special case of the IVP (4.1) when $f$ is the Nemytskij operator associated with the function $q:[a, b] \times \mathbb{R} \times L_{l o c}^{1}(a, b] \rightarrow \mathbb{R}$ by

$$
f(x):=q(\cdot, x(\cdot), x), \quad x \in L_{l o c}^{1}(a, b] .
$$

Considering distributions $\delta_{\lambda}$ as generalized functions $t \alpha \delta(t-\lambda), t \in[a, b]$, we can rewrite the system (4.7), (4.8) as

$$
\frac{d}{d t}(p(t) u(t))=\sum_{\lambda \in \Lambda} I(\lambda, u) \delta(t-\lambda)+q(t, u(t), u) \text { a.e. on }[a, b], \quad \lim _{t \rightarrow a+}(p(t) u(t))=c(u) .
$$

For instance, Proposition 4.1 implies the following result:

Corollary 4.1. The impulsive Cauchy problem (4.9) has the smallest and greatest solutions which are increasing with respect to $q$ and $c$, if $c: L_{l o c}^{1}(a, b] \rightarrow \mathbb{R}$ is increasing and order-bounded, and if the hypotheses (I) and the following hypotheses are valid.

(q0) $q(\cdot, x(\cdot) ; x)$ is Henstock-Kurzweil integrable on $[a, b]$ for every $x \in L_{l o c}^{1}(a, b]$. 
(q1) $K \int_{a}^{t} q(s, x(s), x) d s \leq K \int_{a}^{t} q(s, y(s), y) d s$ for all $t \in[a, b]$ whenever $\times \leq y$ in $L_{l o c}^{1}(a, b]$.

(q2) There exist Henstock-Kurzweil integrable functions $q_{ \pm}:[a, b] \rightarrow \mathbb{R}$ such that $K \int_{a}^{t} q_{-}(s) d s \leq K \int_{a}^{t} q(s, x(s), x) d s \leq K \int_{a}^{t} q_{+}(s) d s$ for all $x \in L_{l o c}^{1}(a, b]$ and $t \in[a, b]$.

Example 4.1. Determine the smallest and greatest solutions of the IVP

$$
t u^{\prime}(t)+u(t)=\frac{1}{10^{4}}\left[10^{4} \arctan (u(1))\right] \delta\left(t-\frac{1}{2}\right)+q(t, u) \text { a.e. on }[0,1], \quad \lim _{t \rightarrow 0+}(t u(t))=0,
$$

when $q$ is defined by

$$
\left\{\begin{array}{c}
q(t, x)=\frac{\left[10^{4} \tanh \left(\int_{\frac{2}{5}}^{1}(x(s) d s)\right]\right.}{10^{4}} h(t), \quad x \in L_{l o c}^{1}(0,1], t \in(0,1], \text { where } \\
h(t)=\left|\cos \left(\frac{1}{t}\right)\right|+\frac{1}{t} \operatorname{sgn}\left(\cos \left(\frac{1}{t}\right)\right) \sin \left(\frac{1}{t}\right), \quad t \in(0,1] .
\end{array}\right.
$$

'[.]' denotes, as before, the greatest integer function, and 'sgn' the sign function.

Solution: The IVP (4.10) is a special case of (4.6), when $a=0, b=1, c(u)=0, p(t)=$ $t, t \in[0,1], I\left(\frac{1}{2}, x\right)=\frac{1}{10^{4}}\left[10^{4} \arctan (x(1))\right]$, and $\Lambda=\left\{\frac{1}{2}\right\}$. The validity of the hypotheses of Corollary 4.1 is easy to verify. Thus, the IVP (4.10) has the smallest and greatest solutions. These solutions are the smallest and greatest fixed points of $G: L_{l o c}^{1}(0,1] \rightarrow L_{l o c}^{1}(0,1]$, defined by

$$
G(x)(t)=\frac{1}{t}\left(\frac{1}{10^{4}}\left[10^{4} \arctan (x(1))\right] H\left(t-\frac{1}{2}\right)+{ }^{K} \int_{0}^{t} q(t, x)\right), \quad x \in L_{l o c}^{1}(0,1], t \in(0,1] .
$$

Calculating the successive approximations

$$
\left\{\begin{array}{l}
y_{n+1}=G\left(y_{n}\right), y_{0}=x_{-} \text {and } z_{n+1}=G\left(z_{n}\right), z_{0}=x_{+}, \text {where } \\
x_{ \pm}(t)= \pm \frac{2}{t} H\left(t-\frac{1}{2}\right)+\frac{1}{2}^{K} \int_{0}^{t} h(s) d s= \pm \frac{2}{t} H\left(t-\frac{1}{2}\right) \pm \frac{1}{t}\left|\cos \left(\frac{1}{t}\right)\right|, t \in(0,1]
\end{array}\right.
$$

it turns out that $\left(y_{n}\right)_{n=0}^{17}$ is strictly increasing, that $\left(z_{n}\right)_{n=0}^{16}$ is strictly decreasing, that $y_{17}=G\left(y_{17}\right)$, and that $z_{16}=G\left(z_{16}\right)$. Thus $u_{*}=y_{17}$ and $u^{*}=z_{16}$ are by Remark 2.1 the smallest and greatest solutions of (4.1) with $c(u)=0$. The exact formulas of $u_{*}$ and $u^{*}$ are

$$
\left\{\begin{array}{l}
u_{*}(t)=-\frac{4439 H\left(t-\frac{1}{2}\right)}{5000 t}-\frac{6313}{10000}\left|\cos \left(\frac{1}{t}\right)\right|, \quad t \in(0,1] \\
u^{*}(t)=\frac{2219 H\left(t-\frac{1}{2}\right)}{2500 t}+\frac{6311}{10000}\left|\cos \left(\frac{1}{t}\right)\right|, \quad t \in(0,1]
\end{array}\right.
$$

Remarks 4.1. The function $(t, x) \propto q(t, x)$, defined in (4.11), has the following properties.

- It is Henstock-Kurzweil integrable, but it is not Lebesgue integrable with respect to the independent variable $t$ if $x \neq 0$, because $h$ is not Lebesgue integrable on $[0,1]$. - Its dependence on the variables $t$ and $x$ is discontinuous, since the signum function sgn, the greatest integer function [.], and the function $h$ are discontinuous.

- Its dependence on the unknown function $x$ is nonlocal, since the integral of function $x$ appears in the argument of the tanh-function. 
- Its dependence on $x$ is not monotone, since $h$ attains positive and negative values in an infinite number of disjoint sets of positive measure. For instance, $y^{*}(t)>y^{*}(t)$ for all $t \in(0,1]$, but the difference function $t \rightarrow q\left(t, y^{*}\right)-q\left(t, y_{*}^{*}\right)$ is neither nonnegative-valued nor Lebesgue integrable on $[0,1]$.

Notice also that in Example 4.1 dependence of the function $I\left(\frac{1}{2}, x\right)=\frac{1}{10^{4}}\left[10^{4} \arctan (x(1))\right]$ on $x$ is discontinuous.

\section{Second order initial value problems}

We shall study the second order initial value problem in this section

$$
\left\{\begin{array}{l}
\left(p \cdot u^{\prime}\right)^{\prime}=f\left(u, u^{\prime}\right), \\
\lim _{t \rightarrow a+}\left(p \cdot u^{\prime}\right)(t)=c\left(u, u^{\prime}\right), \quad \lim _{t \rightarrow a+} u(t)=d\left(u, u^{\prime}\right),
\end{array}\right.
$$

where $f: L_{l o c}^{1}(a, b]^{2} \rightarrow \mathcal{A}_{R}[a, b], c, d: L_{l o c}^{1}(a, b]^{2} \rightarrow \mathbb{R}, p:[a, b] \rightarrow \mathbb{R}_{+},-\infty<a<b<\infty$. We are looking for the smallest and greatest solutions of (5.1) from the set

$$
Y=\left\{u:(a, b] \rightarrow \mathbb{R}: u^{\prime} \in L_{l o c}^{1}(a, b], \lim _{t \rightarrow a+} u(t) \text { and } \lim _{t \rightarrow a+}\left(p \cdot u^{\prime}\right)(t) \text { exist, and } p \cdot u^{\prime} \in \mathcal{R}[a, b]\right\} .
$$

The IVP (5.1) can be converted to a system of integral equations which does not contain derivatives.

Lemma 5.1. Assume that $p:[a, b] \rightarrow \mathbb{R}_{+}$, that $\frac{1}{p} \in L^{1}[a, b]$ and that $f(u, v) \in \mathcal{A}_{R}[a, b]$ for all $u, v \in L_{l o c}^{1}(a, b]$. Then $u$ is a solution of the IVP (5.1) in $Y$ if and only if $\left(u, u^{\prime}\right)=(u, v)$, where $(u, v) \in L_{l o c}^{1}(a, b]^{2}$ is a solution of the system

$$
\left\{\begin{array}{l}
u(t)=d(u, v)+\int_{a}^{t} v(s) d s, \quad t \in(a, b], \\
v(t)=\frac{1}{p(t)}\left(c(u, v)+r \int_{a}^{t} f(u, v)\right), \quad t \in(a, b] .
\end{array}\right.
$$

Proof: Assume that $u$ is a solution of the IVP (5.1) in $Y$, and denote

$$
v(t)=u^{\prime}(t), \quad t \in(a, b] .
$$

The differential equation, the initial conditions of (5.1), the definition (5.2) of $Y$ and the notation (5.4) imply that

$$
\begin{aligned}
r \int_{a}^{t} f(u, v) & =\lim _{r \rightarrow a+} r \int_{r}^{t} f(u, v)=\lim _{r \rightarrow a+} r \int_{r}^{t}(p \cdot v)^{\prime} \\
& \left.=(p \cdot v)(t)-\lim _{r \rightarrow a+}(p \cdot v)(r)\right)=(p \cdot v)(t)-c(u, v), \quad t \in(a, b],
\end{aligned}
$$

and

$$
\begin{aligned}
u(t)-d(u, v) & =\lim _{r \rightarrow a_{+}}(u(t)-u(r))=\lim _{r \rightarrow a_{+}} \int_{r}^{t} u^{\prime}(s) d s \\
& =\int_{a}^{t} u^{\prime}(s) d s=\int_{a}^{t} v(s) d s, \quad t \in(a, b] .
\end{aligned}
$$

Thus, the integral equations of (5.3) hold. 
Conversely, let $(u, v)$ be a solution of the system $(5.3)$ in $L_{l o c}^{1}(a, b]^{2}$. The first equation of (5.3) implies that $u$ is a.e. differentiable and $v=u^{\prime}$, and that the second initial condition of (5.1) is fulfilled. Since $v=u^{\prime}$, it follows from the second equation of (5.3) that

$$
\left(p \cdot u^{\prime}\right)(t)=c\left(u, u^{\prime}\right)+{ }^{r} \int_{a}^{t} f\left(u, u^{\prime}\right), \quad t \in(a, b] .
$$

The equation (5.5) implies that $p \cdot u^{\prime}$ belongs to $\mathcal{R}[a, b]$, and that the differential equation and first initial condition of (5.1) hold. Thus $u$ is a solution of the IVP (5.1) in $Y$.

Assume that $L_{l o c}(a, b]$ is ordered a.e. pointwise, that $Y$ is ordered pointwise, and that the functions $p, f, c$ and $d$ satisfy the following hypotheses:

Our main existence and comparison result for the IVP (5.1) reads as follows.

Theorem 5.1. Assume that $p:[a, b] \rightarrow \mathbb{R}_{+}$, that $\frac{1}{p} \in L^{1}[a, b]$, and that the mappings $f: L_{l o c}^{1}(a, b] \rightarrow \mathcal{A}_{R}[a, b]$ and $c, d: L_{l o c}^{1}(a, b] \rightarrow \mathbb{R}$ are increasing and order-bounded. Then, the IVP (5.1) has the smallest and greatest solutions in $Y$, and they are increasing with respect to $f, c$ and $d$.

Proof: The hypotheses imposed on $f, c$ and $d$ imply that the following conditions are valid.

(f0) $f(u, v)$ is $R P$ integrable on $[a, b]$ for every $(u, v) \in L_{l o c}^{1}(a, b]$, and there exist such $h_{-}, h_{+} \in \mathcal{A}_{R}[a, b]$ that $h_{-} \leqslant f\left(u_{1}, v_{1}\right) \leqslant f\left(u_{2}, v_{2}\right) \leqslant h_{+}$for all $u_{i}, v_{i} \in L_{l o c}^{1}(a, b], i=1,2, u_{1}$ $\leq u_{2}$ and $v_{1} \leq v_{2}$.

(c0) $c_{ \pm} \in \mathbb{R}$, and $c_{-} \leq c\left(u_{1}, v_{1}\right) \leq c\left(u_{2}, v_{2}\right) \leq c_{+}$whenever $u_{i}, v_{i} \in L_{l o c}^{1}(a, b], i=1,2, u_{1} \leq$ $u_{2}$ and $v_{1} \leq v_{2}$.

(d0) $d_{ \pm} \in \mathbb{R}$, and $d_{-} \leq d\left(u_{1}, v_{1}\right) \leq d\left(u_{2}, v_{2}\right) \leq d_{+}$whenever $u_{i}, v_{i} \in L_{\text {loc }}^{1}(a, b], i=1,2, u_{1}$ $\leq u_{2}$ and $v_{1} \leq v_{2}$.

Assume that $P=L_{l o c}^{1}(a, b]^{2}$ is ordered componentwise. We shall first show that the vector-functions $x_{+}, x_{-}$given by

$$
x_{ \pm}(t):=\left(d_{ \pm}+\int_{a}^{t} \frac{1}{p(s)}\left(c_{ \pm}+r \int_{a}^{s} h_{ \pm}\right) d s, \frac{1}{p(t)}\left(c_{ \pm}+r \int_{a}^{t} h_{ \pm}\right)\right)
$$

define functions $x_{ \pm} \in P$. Since $1 / p$ is Lebesgue integrable and the functions $t \mapsto c_{ \pm}+\int_{a}^{t} h_{ \pm}$belong to $\mathcal{R}[a, b]$, then the second components of $x_{ \pm}$belong to $L_{l o c}^{1}(a, b]$. This result implies that the first components of $x_{ \pm}$are defined and continuous, whence they belong to $L_{l o c}^{1}(a, b]$.

Similarly, by applying also the given hypotheses one can verify that the relations

$$
\left\{\begin{array}{l}
G_{1}(u, v)(t):=d(u, v)+{ }^{K} \int_{a}^{t} v(s) d s, t \in(a, b], \\
G_{2}(u, v)(t):=\frac{1}{p(t)}\left(c(u, v)+^{r} \int_{a}^{t} f(u, v)\right), t \in J,
\end{array}\right.
$$

define an increasing mapping $G=\left(G_{1}, G_{2}\right):\left[x_{-}, x_{+}\right] \rightarrow\left[x_{-}, x_{+}\right]$. 
Let $W$ be a well-ordered chain in the range of $G$. The sets $W_{1}=\{u:(u, v) \in W\}$ and $W_{2}=\{v:(u, v) \in W\}$ are well-ordered and order-bounded chains in $L_{l o c}^{1}(a, b]$. It then follows from [[1], Proposition 9.36] that the supremums of $W_{1}$ and $W_{2}$ exist in $L_{l o c}^{1}(a, b]$. Obviously, (sup $W_{1}$, sup $\left.W_{2}\right)$ is the supremum of $W$ in $P$. Similarly one can show that each inversely well-ordered chain of the range of $G$ has the infimum in $P$.

The above proof shows that the operator $G=\left(G_{1}, G_{2}\right)$ defined by (5.7) satisfies the hypotheses of Lemma 2.1, and therefore $G$ has the smallest fixed point $x_{*}=\left(u_{*}, \nu_{*}\right)$ and the greatest fixed point $x^{*}=\left(u^{*}, v^{*}\right)$. It follows from (5.7) that $\left(u_{*}^{*}, v^{*}\right)$ and $\left(u^{*}, v^{*}\right)$ are solutions of the system (5.3). According to Lemma 5.1, $u_{*}$ and $u^{*}$ belong to $Y$ and are solutions of the IVP (5.1).

To prove that $u^{*}$ and $u^{*}$ are the smallest and greatest of all solutions of (5.1) in $Y$, let $u \in Y$ be any solution of (5.1). In view of Lemma 5.1, $(u, v)=\left(u, u^{\prime}\right)$ is a solution of the system (5.3). Applying the hypotheses (f0), (c0) and (d0) it is easy to show that $x$ $=(u, v) \in\left[x_{-}, x_{+}\right]$, where $x_{ \pm}$are defined by (5.6). Thus $x=(u, v)$ is a fixed point of $G=$ $\left(G_{1}, G_{2}\right):\left[x_{-}, x_{+}\right] \rightarrow\left[x_{-}, x_{+}\right]$, defined by (5.7). Because $x_{*}=\left(u_{*}, v_{*}\right)$ and $x^{*}=\left(u^{*}, v^{*}\right)$ are the smallest and greatest fixed points of $G$, then $\left(u_{*}, v_{*}\right) \leq(u, v) \leq\left(u^{*}, v^{*}\right)$. In particular, $u_{*} \leq u \leq u^{*}$, whence $u_{*}$ and $u^{*}$ are the smallest and greatest of all solutions of the IVP (5.1).

The last assertion is an easy consequence of the last conclusion of Lemma 2.1 and the definition (5.7) of $G=\left(G_{1}, G_{2}\right)$.

Consider next the the following special case of (5.1) where the values of $f$ are combined with impulses and a Henstock-Kurzweil integrable function:

$$
f(u, v)(t)=\sum_{\lambda \in \Lambda} I(\lambda, u, v)(\lambda) \delta(t-\lambda)+q(t, u, v), u, v \in L_{l o c}^{1}(a, b], \quad t \in[a, b] .
$$

In this case problem (5.1) can be rewritten as

$$
\left\{\begin{array}{l}
\frac{d}{d t}\left(p(t) u^{\prime}(t)\right)=\sum_{\lambda \in \Lambda} I\left(\lambda, u, u^{\prime}\right) \delta(t-\lambda)+q\left(t, u, u^{\prime}\right) \text { a.e. on }[a, b] \\
\lim _{t \rightarrow a_{+}}\left(p(t) u^{\prime}(t)\right)=c\left(u, u^{\prime}\right), \quad \lim _{t \rightarrow a+} u(t)=d\left(u, u^{\prime}\right) .
\end{array}\right.
$$

The next result is a consequence of Theorem 5.1.

Corollary 5.1. Assume that $p:[a, b] \rightarrow \mathbb{R}_{+}, \frac{1}{p} \in L^{1}[a, b]$, that functions $c, d: L_{l o c}^{1}(a, b]^{2} \rightarrow \mathbb{R}$ are increasing and order-bounded, and that the mappings $q:[a, b] \times L_{l o c}^{1}(a, b]^{2} \rightarrow \mathbb{R}$ and $I: \Lambda \times L_{l o c}^{1}(a, b]^{2} \rightarrow \mathbb{R}$ satisfies the following hypotheses.

$\left(\mathbf{q}_{1}\right) q(\cdot, x)$ is Henstock-Kurzweil integrable on $[a, b]$ for all $x \in L_{l o c}^{1}(a, b]^{2}$.

$\left(\mathbf{q}_{2}\right)$ There exist Henstock-Kurzweil integrable functions $q_{ \pm}:[a, b] \rightarrow \mathbb{R}$ such that ${ }^{K} \int_{a}^{t} q_{-}(s) d s \leq{ }^{K} \int_{a}^{t} q(s, x) d s \leq{ }^{K} \int_{a}^{t} q(s, y) d s \leq{ }^{K} \int_{a}^{t} q_{+}(s) d s, t \in[a, b]$, whenever $\times \leq$ $y$ in $L_{l o c}^{1}(a, b]^{2}$.

(I) $\sum_{\lambda \in \Lambda}|I(\lambda, x)| \leq M<\infty$ for all $x \in L_{\text {loc }}^{1}(a, b]^{2}$, and $\times \alpha I(\lambda, x)$ is increasing when $\lambda \in$ $\Lambda$.

Then, the impulsive IVP (5.8) has the smallest and greatest solutions that are increasing with respect to $q, c$ and $d$. 
Example 5.1. Determine the smallest and greatest solutions of the following singular impulsive IVP.

$$
\left\{\begin{array}{l}
\frac{d}{d t}\left(\sqrt{t} u^{\prime}(t)\right)=\tanh \left(\frac{\left[20 u[1]+10 u^{\prime}[1]\right]}{100}\right) \delta\left(t-\frac{1}{2}\right)+\frac{\left[\int_{1}^{2}\left(u(s)+u^{\prime}(s)\right) d s\right]}{1+\left[\mid \int_{1}^{2}\left(u(s)+u^{\prime}(s)\right]\right.} \frac{d}{d t}\left(t \sin \frac{1}{t}+t\right) \\
\text { a.e. on }[0,3], \quad \lim _{t \rightarrow 0+} \sqrt{t} u^{\prime}(t)=\frac{\left[u^{\prime}(1)\right]}{1+\left|\left[u^{\prime}(1)\right]\right|}, \quad \lim _{t \rightarrow 0+} u(t)=\frac{[u(1)]}{1+\|u(1)\|} .
\end{array}\right.
$$

Solution: System (5.9) is a special case of (5.8) by setting $a=0, b=3, p(t)=\sqrt{t}$, $\Lambda=\left\{\frac{1}{2}\right\}$, and $q, c, d$ and $I$ are given by

$$
\left\{\begin{array}{l}
q(t, u, v)=+\frac{\left[\int_{1}^{2}(u(s)+v(s)) d s\right]}{1+\left|\left[\int_{1}^{2}(u(s)+v(s)) d s\right]\right|} \frac{d}{d t}\left(t \sin \frac{1}{t}+t\right) \\
c(u, v)=\frac{[v(1)]}{1+|[v(1)]|}, d(u, v)=\frac{[u(1)]}{1+|[u(1)]|}, I\left(\frac{1}{2}, u, v\right)=\tanh \left(\frac{[20 u[1]+10 v[1]]}{100}\right)
\end{array}\right.
$$

It is easy to verify that the hypotheses of Corollary 5.1 hold. Thus (5.9) has the smallest and greatest solutions. The functions $x_{-}$and $x_{+}$defined by (5.6) can be calculated, and their first components are:

$$
\begin{aligned}
u_{+}(t)= & 1-\frac{2 \sqrt{2 \pi}}{3}+2 \sqrt{t}+\frac{2 t \sqrt{t}}{3} \sin \frac{1}{t}+\frac{4 \sqrt{t}}{3} \cos \frac{1}{t} \\
& +\frac{4 \sqrt{2 \pi}}{3} \text { Fresnels }\left(\sqrt{\frac{2}{\pi t}}\right)+\frac{2 t \sqrt{t}}{3}+(2 \sqrt{t}-\sqrt{2}) H\left(t-\frac{1}{2}\right), \\
u_{-}= & -u_{+}
\end{aligned}
$$

where

$$
\text { Fresnels }(x)=\int_{0}^{x} \sin \left(\frac{\pi}{2} t^{2}\right) d t
$$

is the Fresnel sine integral. According to Lemma 5.1, the smallest solution of (5.9) is equal to the first component of the smallest fixed point of $G=\left(G_{1}, G_{2}\right)$, defined by (5.7), with $f, c$ and $d$ given by (5.10) and $p(t)=\sqrt{t}$. Calculating the iterations $G^{n} x_{\text {_ }}$ it turns out that $G^{4} x_{-}=G^{5} x_{-}$, whence $G_{1}^{4} x_{-}$is the smallest solution of (5.9). Similarly, one can show that $G_{1}^{3} x_{+}$is the greatest solution of (5.9). The exact expressions of these solutions are

$$
\begin{aligned}
u_{*}(t)= & -\frac{4}{5}+\frac{3 \sqrt{2 \pi}}{5}-\frac{8}{5} \sqrt{t}-\frac{3 t \sqrt{t}}{5} \sin \frac{1}{t}-\frac{6 \sqrt{t}}{5} \cos \frac{1}{t} \\
& -\frac{6 \sqrt{2 \pi}}{5} \text { Fresnels }\left(\sqrt{\frac{2}{\pi t}}\right)-\tanh \left(\frac{11}{10}\right)(2 \sqrt{t}-\sqrt{2}) H\left(t-\frac{1}{2}\right), \\
u^{*}(t)= & \frac{3}{4}-\frac{16 \sqrt{2 \pi}}{27}+\frac{3 \sqrt{t}}{2}+\frac{16 t \sqrt{t}}{27} \sin \frac{1}{t}+\frac{32 \sqrt{t}}{27} \cos \frac{1}{t} \\
& +\frac{32 \sqrt{2 \pi}}{27} \text { Fresnels }\left(\sqrt{\frac{2}{\pi t}}\right)+\frac{4 t \sqrt{t}}{7}+\tanh \left(\frac{21}{20}\right)(2 \sqrt{t}-\sqrt{2}) H\left(t-\frac{1}{2}\right) .
\end{aligned}
$$

\section{Second Order Boundary Value Problems}

This section is devoted to the study of the second order boundary value problem (BVP) 


$$
\left\{\begin{array}{l}
-\left(p \cdot u^{\prime}\right)^{\prime}=f\left(u, u^{\prime}\right) \\
\lim _{t \rightarrow a_{+}}\left(p \cdot u^{\prime}\right)(t)=c\left(u, u^{\prime}\right), \quad u(b)=d\left(u, u^{\prime}\right)
\end{array}\right.
$$

where $f: L^{1}[a, b]^{2} \rightarrow \mathcal{A}_{R}[a, b], c, d: L^{1}[a, b]^{2} \rightarrow \mathbb{R}$, and $p:[a, b] \rightarrow \mathbb{R}_{+},-\infty<a<b<\infty$. Now we are looking for the smallest and greatest solutions of (6.1) from the set

$$
Z=\left\{u:(a, b] \rightarrow \mathbb{R}: u^{\prime} \in L^{1}[a, b], \lim _{t \rightarrow a+}\left(p \cdot u^{\prime}\right)(t) \text { exists, and } p \cdot u^{\prime} \in \mathcal{R}[a, b]\right\} .
$$

The BVP (6.1) can be transformed into a system of integral equations as follows.

Lemma 6.1. Assume that $p:[a, b] \rightarrow \mathbb{R}_{+}$, that $\frac{1}{p} \in L^{1}[a, b]$, and that $f(u, v) \in \mathcal{A}_{R}[a, b]$ for all $u, v \in L^{1}[a, b]$. Then $u$ is a solution of the IVP (6.1) in $Z$ if and only if $\left(u, u^{\prime}\right)=(u, v)$, where $(u, v) \in L^{1}[a, b]^{2}$ is a solution of the system

$$
\left\{\begin{array}{l}
u(t)=d(u, v)-\int_{t}^{b} v(s) d s, \quad t \in[a, b], \\
v(t)=\frac{1}{p(t)}\left(c(u, v)-r \int_{a}^{t} f(u, v)\right), \quad t \in[a, b],
\end{array}\right.
$$

Proof: Assume that $u$ is a solution of the BVP (6.1) in $Z$, and denote

$$
v(t)=u^{\prime}(t), \quad t \in[a, b] .
$$

The differential equation, the boundary conditions of (6.1), the definition (6.2) of $Z$ and the notation (6.4) ensure that

$$
\begin{aligned}
-{ }^{r} \int_{a}^{t} f(u, v) & =-\lim _{r \rightarrow a+} r \int_{r}^{t} f(u, v)=\lim _{r \rightarrow a+} r \int_{r}^{t}(p \cdot v)^{\prime} \\
& \left.=\lim _{r \rightarrow a+}(p \cdot v)(t)-(p \cdot v)(r)\right)=(p \cdot v)(t)-c(u, v), \quad t \in[a, b],
\end{aligned}
$$

and

$$
u(t)-d(u, v)=u(t)-u(b)=-\int_{t}^{b} u^{\prime}(s) d s=-\int_{t}^{b} v(s) d s, \quad t \in[a, b] .
$$

Thus the integral equations of (6.3) hold.

Conversely, let $(u, v)$ be a solution of the system (6.3) in $L^{1}[a, b]^{2}$. The first equation of (6.3) implies that $u$ is a.e. differentiable and $v=u^{\prime}$, and that the second boundary condition of (6.1) holds. Since $v=u^{\prime}$, it follows from the second equation of (6.3) that

$$
\left(p \cdot u^{\prime}\right)(t)=c\left(u, u^{\prime}\right)-r \int_{a}^{t} f\left(u, u^{\prime}\right), \quad t \in[a, b] .
$$

This equation implies that $p \cdot u^{\prime}$ belongs to $\mathcal{R}[a, b]$, and that the differential equation and first boundary condition of (6.1) are satisfied. Thus $u$, is a solution of the BVP (6.1) in $Z$. $\square$

Assume that $L^{1}[a, b]$ is ordered a.e. pointwise, that $Z$ is ordered pointwise. We shall impose the following hypotheses for the functions $p, f, c$, and $d$.

$\left(\mathbf{p}_{1}\right) p:[a, b] \rightarrow \mathbb{R}_{+}$, and $\frac{1}{p} \in L^{1}[a, b]$.

$\left(\mathbf{f}_{1}\right) f: L^{1}[a, b]^{2} \rightarrow \mathcal{A}_{R}[a, b]$ is order-bounded, and $f\left(u_{1}, v_{1}\right) \preccurlyeq f\left(u_{2}, v_{2}\right)$ whenever $u_{i}$, $v_{i} \in L^{1}[a, b], i=1,2, u_{1} \leq u_{2}$, and $v_{1} \geq v_{2}$. 
$\left(\mathbf{c}_{1}\right) c: L^{1}[a, b]^{2} \rightarrow \mathbb{R}$ is order-bounded, and $c\left(u_{2}, v_{2}\right) \leq c\left(u_{1}, v_{1}\right)$ whenever $u_{i}, v_{i} \in L^{1}$ $[a, b], i=1,2, u_{1} \leq u_{2}$, and $v_{1} \geq v_{2}$.

$\left(\mathbf{d}_{1}\right) d: L^{1}[a, b]^{2} \rightarrow \mathbb{R}$ is order-bounded, and $d\left(u_{1}, v_{1}\right) \leq d\left(u_{2}, v_{2}\right)$ whenever $u_{i}, v_{i} \in$ $L^{1}[a, b], i=1,2, u_{1} \leq u_{2}$ and $v_{1} \geq v_{2}$.

The next theorem is our main existence and comparison result for the BVP (6.1).

Theorem 6.1. Assume that the hypotheses $\left(p_{1}\right),\left(f_{1}\right),\left(c_{1}\right)$, and $\left(d_{1}\right)$ hold. Then, the BVP (6.1) has the smallest and greatest solutions in $Z$, and they are increasing with respect to $f$ and $d$ and decreasing with respect to $c$.

Proof: Because $f, c$ and $d$ are order-bounded, then the following conditions are valid.

$\left(\mathbf{f}_{0}\right)$ There exist $h_{ \pm} \in \mathcal{A}_{R}[a, b]$ such that $h_{-} \preccurlyeq f(u, v) \preccurlyeq h_{+}$for all $u, v \in L^{1}[a, b]$.

$\left(\mathbf{c}_{0}\right)$ There exist $c_{ \pm} \in \mathbb{R}$ such that $c_{-} \leq c(u, v) \leq c_{+}$whenever $u, v \in L^{1}[a, b]$.

$\left(\mathbf{d}_{0}\right)$ There exist $d_{ \pm} \in \mathbb{R}$ such that $d_{-} \leq d(u, v) \leq d_{+}$whenever $u, v \in L^{1}[a, b]$.

Assume that $P=L^{1}[a, b]^{2}$ is ordered by

$$
\left(u_{1}, v_{1}\right) \leq\left(u_{2}, v_{2}\right) \text { if and only if } u_{1} \leq u_{2} \text {, and } v_{1} \geq v_{2} \text {. }
$$

We shall first show that the vector-functions $x_{+}, x_{-}$given by

$$
\left\{\begin{array}{l}
x_{-}(t)=\left(d_{-}-\int_{t}^{b} \frac{1}{p(s)}\left(c_{+}-{ }^{r} \int_{a}^{s} h_{-}\right) d s, \frac{1}{p(t)}\left(c_{+}-{ }^{r} \int_{a}^{t} h_{-}\right)\right), \\
x_{+}(t)=\left(d_{+}-\int_{t}^{b} \frac{1}{p(s)}\left(c_{-}-r \int_{a}^{s} h_{+}\right) d s, \frac{1}{p(t)}\left(c_{-}-{ }^{r} \int_{a}^{t} h_{+}\right)\right) .
\end{array}\right.
$$

belong to $P$. Since $1 / p$ is Lebesgue integrable and the function $t \mapsto c_{+}-{ }^{r} \int_{a}^{t} h_{-}$ belongs to $\mathcal{R}[a, b]$, then the second component of $x_{+}$is Lebesgue integrable on $[a, b]$.

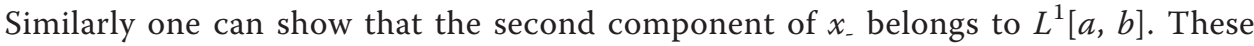
results ensure that the first components of $x_{ \pm}$are defined and continuous in $t$, and hence are in $L^{1}[a, b]$.

Similarly, by applying the given hypotheses one can verify that the relations

$$
\left\{\begin{array}{c}
G_{1}(u, v)(t):=d(u, v)-{ }^{K} \int_{t}^{b} v(s) d s, t \in[a, b], \\
G_{2}(u, v)(t):=\frac{1}{p(t)}\left(c\left(u, v^{r} \int_{a}^{t} f(u, v)\right)\right), \quad t \in[a, b]
\end{array}\right.
$$

define an increasing mapping $G=\left(G_{1}, G_{2}\right):\left[x_{-}, x_{+}\right] \rightarrow\left[x_{-}, x_{+}\right]$.

Let $W$ be a well-ordered chain in the range of $G$. The set $W_{1}=\{u:(u, v) \in W\}$ is well ordered, $W_{2}=\{v:(u, v) \in W\}$ is inversely well-ordered, and both $W_{1}$ and $W_{2}$ are order-bounded in $L^{1}[a, b]$. It then follows from [1, Lemma 9.32] that the supremum of $W_{1}$ and the infimum of $W_{2}$ exist in $L^{1}[a, b]$. Obviously, (sup $W_{1}$, inf $\left.W_{2}\right)$ is the supremum of $W$ in $(P, \leq)$. Similarly, one can show that each inversely well-ordered chain of the range of $G$ has the infimum in $(P, \leq)$.

The above proof shows that the operator $G=\left(G_{1}, G_{2}\right)$ defined by (6.8) satisfies the hypotheses of Lemma 2.1 , whence $G$ has the smallest fixed point $x_{*}=\left(u_{*}, v_{*}\right)$ and a greatest fixed point $x^{*}=\left(u^{*}, v^{*}\right)$. It follows from (6.8) that $\left(u_{*}, v_{*}\right)$ and $\left(u^{*}, v^{*}\right)$ are solutions of the system (6.3). According to Lemma 6.1, $u_{*}$ and $u^{*}$ belong to $Z$ and are solutions of the BVP (6.1).

To prove that $u^{*}$ and $u^{*}$ are the smallest and greatest of all solutions of (6.1) in $Z$, let $u \in Z$ be any solution of (6.1). In view of Lemma $6.1,(u, v)=\left(u, u^{\prime}\right)$ is a solution of 
the system (6.3). Applying the properties $\left(f_{0}\right),\left(c_{0}\right)$, and $\left(d_{0}\right)$ it is easy to show that $x=$ $(u, v) \in\left[x_{-}, x_{+}\right]$, where $x_{ \pm}$are defined by (6.7). Thus, $x=(u, v)$ is a fixed point of $G=$ $\left(G_{1}, G_{2}\right):\left[x_{-}, x_{+}\right] \rightarrow\left[x_{-}, x_{+}\right]$, defined by (6.8). Because $x_{*}=\left(u_{*}, v_{*}\right)$ and $x^{*}=\left(u^{*}, v^{*}\right)$ are the smallest and greatest fixed points of $G$, respectively, then $\left(u_{*}, v_{*}\right) \leq(u, v) \leq\left(u^{*}, v^{*}\right)$. In particular, $u_{*} \leq u \leq u^{*}$, whence $u_{*}$ and $u^{*}$ are the smallest and greatest of all solutions of the BVP (6.1).

The last assertion is an easy consequence of the last conclusion of Lemma 2.1, and the definition (6.8) of $G=\left(G_{1}, G_{2}\right)$. $\square$

Consider next a special case of (6.1) where the values of $f$ combined with impulses and Henstock-Kurzweil integrable functions:

$$
\left\{\begin{array}{l}
\frac{d}{d t}\left(p(t) u^{\prime}(t)\right)=\sum_{\lambda \in \Lambda} \alpha(\lambda) \delta(t-\lambda)+g\left(u, u^{\prime}\right)(t) \text { a.e. on }[a, b] \\
\lim _{t \rightarrow a+}\left(p(t) u^{\prime}(t)\right)=c\left(u, u^{\prime}\right), \quad u(b)=d\left(u, u^{\prime}\right)
\end{array}\right.
$$

Corollary 6.1. Assume that $p:[a, b] \rightarrow \mathbb{R}_{+}, \frac{1}{p} \in L^{1}[a, b]$, that functions $c, d: L^{1}[a, b]^{2}$ $\rightarrow \mathbb{R}$ satisfy the hypotheses $\left(c_{i}\right)$ and $\left(d_{i}\right), i=1,2$, that $\alpha: \Lambda \rightarrow \mathbb{R}, \sum_{\lambda \in \Lambda} \mid \alpha(\lambda \mid<\infty$, and that $g$ satisfies the following hypotheses.

$\left(\mathbf{g}_{1}\right) g(u, v)$ is Henstock-Kurzweil integrable on $[a, b]$ for all $u, v \in L^{1}[a, b]$.

$\left(\mathbf{g}_{2}\right)$ There exist Henstock-Kurzweil integrable functions $\underline{g}^{\prime} \bar{g}:[a, b] \rightarrow$ Rsuch that $K \int_{a}^{t} \underline{g} \leq K \int_{a}^{t} g\left(u_{1}, v_{1}\right) \leq K \int_{a}^{t} g\left(u_{2}, v_{2}\right) \leq K \int_{a}^{t} \bar{g}, t \in[a, b]$, whenever $u_{1} \leq u_{2}$ and $v_{1}$ $\geq v_{2}$ in $L^{1}[a, b]$.

Then, the impulsive BVP (6.9) has the smallest and greatest solutions that are increasing with respect to $g, d$ and decreasing with respect to $c$.

Example 6.1. Determine the smallest and greatest solutions of the following singular impulsive BVP.

$$
\left\{\begin{array}{l}
-\frac{d}{d t}\left(\sqrt{t} u^{\prime}(t)\right)=\delta\left(t-\frac{3}{2}\right)+\frac{d}{d t}\left(t \sin \frac{1}{t}\right) \tanh \left(\frac{1}{1000}\left[\int_{1}^{2}\left(3000 u(s)-2000 u^{\prime}(s)\right) d s\right]\right) \\
\text { a.e. on }[0,3], \quad \lim _{t \rightarrow 0+} \sqrt{t} u^{\prime}(t)=\frac{\left[1000 u^{\prime}(1)\right]}{1+\left.\left[1000 u^{\prime}(1)\right]\right|^{\prime}}, \quad u(3)=\frac{[1000 u(1)]}{1+\mid[1000 u(1) \|} .
\end{array}\right.
$$

Solution: System (6.10) is a special case of (6.9) when $a=0, b=3$, $\Lambda=\left(\frac{3}{2}\right) \alpha\left(\frac{3}{2}\right)=1, \Lambda=\left(\frac{3}{2}\right)$, and $g, c, d$ are given by

$$
\left\{\begin{array}{l}
g(u, v)(t)=\delta\left(t-\frac{3}{2}\right)+\frac{d}{d t}\left(t \sin \frac{t}{t}\right) \tanh \left(\frac{1}{1000}\left[\int_{1}^{2}(3000 u(s)-2000 v(s)) d s\right]\right) \\
c(u, v)=\frac{[1000 v(1)]}{1+|[1000 v(1)]|}, d(u, v)=\frac{[1000 u(1)]}{1+|[1000 u(1)]|}
\end{array}\right.
$$

It is easy to verify that the hypotheses of Corollary 6.1 are valid. Thus (6.10) has the smallest and greatest solutions. The functions $x_{-}$and $x_{+}$defined by (6.7) can be calculated, and their first components are:

$$
u_{-}(t)=\left\{\begin{array}{l}
-1-\sqrt{6}-2 \sqrt{3}-2 \sqrt{3} \sin \frac{1}{3}-\frac{4}{3} \sqrt{3} \cos \frac{1}{3}-\frac{4 \sqrt{2 \pi}}{3} \text { Fresnels }\left(\frac{\sqrt{6}}{3 \sqrt{\pi}}\right)+2 \sqrt{t} \\
+\frac{2 t \sqrt{t}}{3} \sin \frac{1}{t}+\frac{4 \sqrt{t}}{3} \cos \frac{1}{t}+\frac{4 \sqrt{2 \pi}}{3} \text { Fresnels }\left(\frac{\sqrt{2}}{\sqrt{t \pi}}\right)+\frac{20 t \sqrt{t}}{3}-(2 \sqrt{t}-\sqrt{6}) H\left(t-\frac{1}{3}\right)
\end{array}\right.
$$


and

$$
u_{+}(t)\left\{\begin{array}{l}
1-\sqrt{6}+6 \sqrt{3}+2 \sqrt{3} \sin \frac{1}{3}+\frac{4 \sqrt{3}}{3} \cos \frac{1}{3}+\frac{4 \sqrt{2 \pi}}{3} \text { Fresnels }\left(\frac{\sqrt{6}}{3 \sqrt{\pi}}\right)-2 \sqrt{t} \\
-\frac{2 t \sqrt{t}}{3} \sin \frac{1}{t}-\frac{4 \sqrt{t}}{3} \cos \frac{1}{t}-\frac{4 \sqrt{2 \pi}}{3} \text { Fresnels }\left(\frac{\sqrt{2}}{\sqrt{t \pi}}\right)-\frac{2 t \sqrt{t}}{3}-(2 \sqrt{t}-\sqrt{6}) H\left(t-\frac{1}{3}\right),
\end{array}\right.
$$

where FresnelS is the Fresnel sine integral.

According to Lemma 6.1 the smallest solution of (6.10) is equal to the first component of the smallest fixed point of $G=\left(G_{1}, G_{2}\right)$, defined by (6.3). Calculating the first iterations $G^{n} x_{-}$it turns out that $G^{6} x_{-}=G^{7} x_{-}$. Thus $G_{1}^{6} x_{-}$is the smallest solution of (6.10). Similarly, one can show that $G^{3} x_{+}=G^{4} x_{+}$, whence $G_{1}^{3} x_{+}$is the greatest solution of (6.10). The exact expressions of these solutions are

$$
u_{*}(t)=\left\{\begin{array}{l}
-\frac{5623}{5624}-\frac{1}{1421} \sqrt{3}-2 \sqrt{3} \tanh \frac{3559}{200}\left(1+\sin \frac{1}{3}\right)-\sqrt{6}-\frac{4 \sqrt{3}}{3} \tanh \frac{3559}{200} \cos \frac{1}{3} \\
+\frac{4 \sqrt{2 \pi}}{3} \tanh \frac{3559}{200} \text { Fresnels }\left(\frac{\sqrt{6}}{3 \sqrt{\pi}}\right)+\frac{2841 \sqrt{t}}{1421}+\frac{2 t \sqrt{t}}{3} \tanh \frac{3559}{200}\left(\sin \frac{1}{t}+1\right)+\frac{4 \sqrt{t}}{3} \tanh \frac{3559}{200} \cos \frac{1}{t} \\
-\frac{4 \sqrt{2 \pi}}{3} \tanh \frac{3559}{200} \text { Fresnels }\left(\frac{\sqrt{2}}{\sqrt{t \pi}}\right)+\frac{2 t \sqrt{t}}{3} \tanh \frac{3559}{200}-(2 \sqrt{t}-\sqrt{6}) H\left(t-\frac{1}{3}\right),
\end{array}\right.
$$

and

$$
u^{*}(t)=\left\{\begin{array}{l}
-\frac{7652}{7653}-\frac{11370}{2843} \sqrt{3}+2 \sqrt{3} \tanh \frac{4963}{200}\left(1+\sin \frac{1}{3}\right)-\sqrt{6}+\frac{4 \sqrt{3}}{3} \tanh \frac{4963}{200} \cos \frac{1}{3} \\
+\frac{4 \sqrt{2 \pi}}{3} \tanh \frac{4963}{200} \text { Fresnels }\left(\frac{\sqrt{6}}{3 \sqrt{\pi}}\right)-\frac{5684 \sqrt{t}}{2843}-\frac{2 t \sqrt{t}}{3} \tanh \frac{4963}{200}\left(\sin \frac{1}{t}+1\right)-\frac{4 \sqrt{t}}{3} \tanh \frac{4963}{200} \cos \frac{1}{t} \\
-\frac{4 \sqrt{2 \pi}}{3} \tanh \frac{4963}{200} \text { Fresnels }\left(\frac{\sqrt{2}}{\sqrt{t \pi}}\right)+\frac{2 t \sqrt{t}}{3} \tanh \frac{3559}{200}-(2 \sqrt{t}-\sqrt{6}) H\left(t-\frac{1}{3}\right),
\end{array}\right.
$$

Remarks 6.1. The IVP's (3.1) and (5.1) and the BVP (6.1) can be

- singular, since $\lim _{t \rightarrow a+} p(t)=0$ is allowed;

- nonlocal, because the functions $g, c, d$, and $f$ may depend functionally on $u$ and/ or $u^{\prime}$;

- discontinuous, since the dependencies of $g, c, d$ and $f$ on $u$ and/or $u$ ' can be discontinuous;

- distributional, since the values of $g$ and $f$ can be distributions;

- impulsive, since the values of $g$ and $f$ can contain impulses.

A theory for first order nonlinear distributional Cauchy problems is presented in [12]. Linear distributional differential equations are studied in $[13,8]$. Singular ordinary differential equations are studied, e.g., in $[11,14,15]$. Initial value problems in ordered Banach spaces are studied, e.g., in [1-4,7]. As for the study of impulsive differential equations, see, e.g. $[1,16,17]$. The case of well-ordered set of impulses is studied first time in [18].

The solutions of examples have been calculated by using simple Maple programming.

Acknowledgements

The author thanks the anonymous referee for a careful review and constructive comments.

Authors' contributions

The work was realized by the author.

Competing interests

The author declares that they have no competing interests. 


\section{References}

1. Carl, S, Heikkilä, S: Fixed Point Theory in Ordered Spaces and Applications. Springer, Berlin (2011)

2. Heikkilä, S, Kumpulainen, M: On improper integrals and differential equations in ordered Banach spaces. J Math Anal Appl. 319, 579-603 (2006). doi:10.1016/j.jmaa.2005.06.051

3. Heikkilä, S, Kumpulainen, M: Differential equations with non-absolutely integrable functions in ordered Banach spaces. Nonlinear Anal. 72, 4082-4090 (2010). doi:10.1016/j.na.2010.01.040

4. Heikkilä, S, Kumpulainen, M: Second order initial value problems with non-absolute integrals in ordered Banach spaces. Nonlinear Anal. 74(5), 1939-1955 (2011)

5. Talvila, E: The regulated primitive integral. III J Math. 53, 1187-1219 (2009)

6. Talvila, E: The distributional Denjoy integral. Real Anal Exch. 33, 51-82 (2008)

7. Heikkilä, S, Lakshmikantham, V: Monotone Iterative Techniques for Discontinuous Nonlinear Differential Equations. Marcel Dekker Inc., New York (1994)

8. Tvrdý, M: Linear distributional differential equations of the second order. Math Bohemica. 119(4), 419-436 (1994)

9. Friedlander, FG, Joshi, M: Introduction to the Theory of Distributions. Cambridge University Press, Cambridge (1999)

10. Schwartz, L: Théorie des distributions. Hermann, Paris (1966)

11. Carl, S, Heikkilä, S: Nonlinear Differential Equations in Ordered Spaces. Chapman \& Hall/CRC, Boca Raton (2000)

12. Heikkilä, S: On nonlinear distributional and impulsive Cauchy problems. (in press)

13. Pelant, M, Tvrdý, M: Linear distributional differential equations in the space of regulated functions. Math Bohemica. 118(4), 379-400 (1993)

14. Heikkilä, S, Seikkala, S: On the existence of extremal solutions of phi-Laplacian initial and boundary value problems. Int J Pure Appl Math. 17(1), 119-138 (2004)

15. Heikkilä, S, Seikkala, S: On singular, functional, nonsmooth and implicit phi-Laplacian initial and boundary value problems. J Math Anal Appl. 308(2), 513-531 (2005). doi:10.1016/j.jmaa.2004.11.033

16. Federson, M, Táboas, P: Impulsive retarded differential equations in Banach spaces via Bochner-Lebesgue and Henstock integrals. Nonlinear Anal. 50, 389-407 (2002). doi:10.1016/S0362-546X(01)00769-6

17. Lakshmikantham, V, Bainov, D, Simeonov, P: Theory of Impulsive Differential Equations, World Scientific, Singapore. (1989)

18. Heikkilä, S, Kumpulainen, M, Seikkala, S: Uniqueness and existence results for implicit impulsive differential equations. Nonlinear Anal. 42, 13-26 (2000), doi:10.1016/S0362-546X(98)00325-3

doi:10.1186/1687-2770-2011-24

Cite this article as: Heikkilä: On singular nonlinear distributional and impulsive initial and boundary value problems. Boundary Value Problems 2011 2011:24.

\section{Submit your manuscript to a SpringerOpen ${ }^{\circ}$ journal and benefit from:}

Convenient online submission

Rigorous peer review

- Immediate publication on acceptance

- Open access: articles freely available online

- High visibility within the field

- Retaining the copyright to your article

Submit your next manuscript at $\boldsymbol{s p r i n g e r o p e n . c o m ~}$ 